\title{
On the functional representation of partition function for quantum magnetic cluster systems
}

\author{
N.A.Korynevskii ${ }^{1,2}$ \\ 1 Institute for Condensed Matter Physics \\ of the National Academy of Sciences of Ukraine, \\ 1 Svientsitskii Str., 79011 Lviv, Ukraine \\ 2 Institute of Physics, University of Szczecin, \\ 15 Wielkopolska Str., 70451 Szczecin, Poland
}

Received February 6, 2002, in final form June 13, 2002

The problem of the functional representation for systems containing groups of atoms with a non-compensated spin momentum (magnetic clusters) is discussed. For representation of the functional of partition function a version of the collective variables method with the "reference system" as a zero-order approximation is used. A set of all isolated clusters are choosen as a reference system. Intracluster interactions are described by exchange Heisenberg-type Hamiltonian, the form of intercluster interactions depend on the structure of the system investigated. Due to the use of the recently introduced generalized transition operators (like well-known HubbardStasyuk operators) an explicit form of the functional of partition function is found.

Key words: cluster system, functional of partition function, reference system

PACS: $05.60 .+W, 05.70 . \mathrm{Ln}, 05.20 . \mathrm{Dd}, 52.25 . \mathrm{Dg}, 52.25 . \mathrm{Fi}$

\section{Introduction}

Among different kinds of physical systems, in which the processes of structural elements ordering play an essential role, a cluster system accupies a special place. In a crystalline (or amorphous) cluster system, due to its internal structure or peculiarities of interparticle interactions, there are physically distinguished groups of particles. So, the correlations between the particles from different groups are much weaker as compared with analogous correlations of the particles belonging to the same group. One may divide magnetic cluster systems into two classes. To the first class there belong natural pure compounds, for example $\left[\mathrm{Cr}_{3}\left(\mathrm{CH}_{3} \mathrm{COO}\right)_{6}(\mathrm{OH})_{2}\right] \mathrm{Cl} \cdot 8 \mathrm{H}_{2} \mathrm{O}$, $\left[\mathrm{Fe}_{3}\left(\mathrm{CH}_{3} \mathrm{COO}\right)_{6}(\mathrm{OH})_{2}\right] \mathrm{NO}_{3} \cdot 6 \mathrm{H}_{2} \mathrm{O}$ (three-particle clusters), $\mathrm{Cu}_{2}\left(\mathrm{CH}_{3} \mathrm{COO}\right)_{2} \cdot 2 \mathrm{H}_{2} \mathrm{O}$ 
(two-particle clusters). The second class is formed of artificially prepared specimen, for example a solid mixture of a magnetic compound $\mathrm{MnO}$ in a diamagnetic matrix $\mathrm{MgO}$ (large set of isolated ions, two-, three- and more particles clusters) [1]. It should be noted that the cluster structure is a characteristic feature for different ferroelectric compounds: DMAAS and DMAGaS [2], SASD and SASeD [3] and some others.

The theoretical investigations of the magnetic cluster systems known at the present time are devoted only to the description of statistic properties and thermodynamic functions of isolated small clusters (two-, three- or four particles) on the Heisenberg model basis. The intercluster interactions in this descriptions are neglected but they play an essential role in the cluster system behaviour at the phase transition point neighbourhood.

As far as a magnetic moment in all the mentioned cluster systems is of a spin nature, the usage of the Heisenberg-type Hamiltonian for exchange interaction description is quite adequate. But exchange interactions are essential only for the particles belonging to the same cluster (due to a small distance between particles). Besides the exchange interaction in magnets, there exist dipole-dipole magnetic interparticle interactions and interactions of magnetic atoms with crystalline lattice electric field. Due to its relativistic nature, the last two interactions are 1-2 orders weaker than the exchange interactions. But their role is very essential because they are responsible for the formation of the axes of magnetization and possess a large radius of action [4]. Therefore, it is nessesary to take into account magnetic dipole-dipole interactions for a correct description of the intercluster correlations.

The main purpose of this paper is to get a functional representation for a partition function of the interacting magnetic cluster system. The intracluster interactions are described by quantum Heisenberg-type Hamiltonian. The intercluster correlations appear due to taking into account the dipole-dipole pair interactions between particles. The general approach to the calculation is based on the collective variables method [5] and on the usage of the generalized transition operators [6]. The obtained representation will be used for a rigorous description of the ferromagnetic cluster systems behaviour in the phase transition point neighbourhood.

\section{Hamiltonian. Reference system}

Three-dimensional crystalline lattice, containing $f_{0}$ spin particles (atoms with non-compensated spin momentum, or electrons) at each of the $N$ cells is considered. In the spin operators representation such a system is described by the following Hamiltonian:

$$
\begin{aligned}
H= & -\sum_{q=1}^{N} \sum_{f=1}^{f_{0}} h_{f}\left(\vec{R}_{q}\right) S_{f}^{z}\left(\vec{R}_{q}\right)-2 \sum_{q=1}^{N} \sum_{f, f^{\prime}=1}^{f_{0}} V_{f f^{\prime}} \vec{S}_{f}\left(\vec{R}_{q}\right) \vec{S}_{f^{\prime}}\left(\vec{R}_{q}\right) \\
& -\frac{1}{2} \sum_{\alpha} \sum_{q, q^{\prime}=1}^{N} \sum_{f, f^{\prime}=1}^{f_{0}} J_{f f^{\prime}}^{\alpha \alpha}\left(\vec{R}_{q}, \vec{R}_{q}^{\prime}\right) S_{f}^{\alpha}\left(\vec{R}_{q}\right) S_{f^{\prime}}^{\alpha}\left(\vec{R}_{q^{\prime}}\right) .
\end{aligned}
$$


Here $S_{f}^{\alpha}\left(\vec{R}_{q}\right)$ is the $\alpha$-component of the $f$-th spin particle situated in the $q$ cell, $V_{f f^{\prime}}$ is the exchange integral, $J_{f f^{\prime}}^{\alpha \alpha}\left(\vec{R}_{q}, \vec{R}_{q^{\prime}}\right)$ is a coefficient of the magnetic dipole-dipole interaction between $\alpha$ components of spins from different cells, $h_{f}\left(\vec{R}_{q}\right)$ is the external magnetic field.

Two first terms in (2.1) belong to the particles situated at the same cell (in the cluster) and $V_{f f^{\prime}} \gg J_{f f^{\prime}}^{\alpha \alpha}\left(\vec{R}_{q}, \vec{R}_{q^{\prime}}\right)$. It is natural to consider such groups of particles in (2.1) as a reference system. Putting $V_{f f^{\prime}}=V$ (topologically it corresponds to the clusters of 2,3 or 4 particles only) and $h_{f}\left(\vec{R}_{q}\right)=h$ (a uniform external field), one obtains the expression for reference system Hamiltonian:

$$
H_{0}=-h \sum_{q=1}^{N} \sum_{f=1}^{f_{0}} S_{f}^{z}\left(\vec{R}_{q}\right)-2 V \sum_{q=1}^{N} \sum_{f, f^{\prime}=1}^{f_{0}} \vec{S}_{f}\left(\vec{R}_{q}\right) \vec{S}_{f^{\prime}}\left(\vec{R}_{q}\right) .
$$

Taking into account the relation for a finite sum of spin operators products:

$$
-2 \sum_{f<f^{\prime}=1}^{f_{0}} \vec{S}_{f} \vec{S}_{f^{\prime}}=\sum_{f=1}^{f_{0}} \vec{S}_{f}^{2}-\left(\vec{S}^{\prime}\right)^{2}
$$

where $\vec{S}$ is a total spin of $f_{0}$ spin particles, one can easily get a formula for an energy spectrum of $f_{0}$-particles cluster, which is described by the Hamiltonian (2.2):

$$
\begin{aligned}
& E=V\left[f_{0} S(S+1)-S^{\prime}\left(S^{\prime}+1\right)\right]-M^{\prime} h, \\
& S^{\prime}=0,1,2,3, \ldots, f_{0} S \quad \text { when } f_{0} \text { is even, } \\
& S^{\prime}=\frac{1}{2}, \frac{3}{2}, \frac{5}{2}, \ldots, f_{0} S \quad \text { when } f_{0} \text { is odd, } \\
& M^{\prime}=-S^{\prime},-S^{\prime}+1,-S^{\prime}+2, \ldots, S^{\prime}-2, S^{\prime}-1, S^{\prime},
\end{aligned}
$$

are possible eigenvalues of the total spin and its projection on the $z$-axis for $S=\frac{1}{2}$.

Therefore, for clusters:

$$
\begin{aligned}
& \underline{f_{0}=2} \quad \frac{E_{1}}{N}=\frac{3}{2} V, \quad \frac{E_{2}}{N}=-\frac{1}{2} V+h, \quad \frac{E_{3}}{N}=-\frac{1}{2} V, \quad \frac{E_{4}}{N}=-\frac{1}{2} V-h ; \\
& \underline{f_{0}=3} \quad \frac{E_{1}}{N}=\frac{3}{2} V+\frac{1}{2} h, \quad \frac{E_{2}}{N}=\frac{3}{2} V-\frac{1}{2} h, \quad \frac{E_{3}}{N}=-\frac{3}{2} V+\frac{3}{2} h, \\
& \frac{E_{4}}{N}=-\frac{3}{2} V+\frac{1}{2} h, \quad \frac{E_{5}}{N}=-\frac{3}{2} V-\frac{1}{2} h, \quad \frac{E_{6}}{N}=-\frac{3}{2} V-\frac{3}{2} h ; \\
& \underline{f_{0}=4} \quad \frac{E_{1}}{N}=3 V, \quad \frac{E_{2}}{N}=V+h, \quad \frac{E_{3}}{N}=V, \quad \frac{E_{4}}{N}=V-h, \quad \frac{E_{5}}{N}=-3 V+2 h, \\
& \frac{E_{6}}{N}=-3 V+h, \quad \frac{E_{7}}{N}=-3 V, \quad \frac{E_{8}}{N}=-3 V-h, \quad \frac{E_{9}}{N}=-3 V-2 h .
\end{aligned}
$$

So, the formula (2.4) gives exact values for energy levels of the cluster. But some levels remain degenerate (starting from $f_{0}=3$ ), because the total number of states is equal to

$$
n=2^{f_{0}} \text {. }
$$


The problem of complete determination of quantum states and corresponding energy levels may be solved using a clusterization method by means of the generalized transition operators. This enables one to calculate the partition function of the reference system. The main purpose of the method of clusterization developed in [6,7] was to obtain a diagonal and symmetrized form for de Gennes-type Hamiltonian. This becomes possible due to the use of the generalized transition operators

$$
Y_{\lambda}\left(\vec{R}_{q}\right)=\sum_{i, j} U_{\lambda_{i j}} X^{i j}\left(\vec{R}_{q}\right)
$$

where Hubbard-Stasyuk operators $X^{i j}\left(\vec{R}_{q}\right)[8,9]$ were constructed on the operators of $z$-component of a total spin of cluster, so, for $H_{0}$ Hamiltonian the next representation took place

$$
H_{0}=\sum_{q=1}^{N} \sum_{i=1}^{2^{f_{0}}} a_{i} X^{i i}\left(\vec{R}_{q}\right)
$$

( $a_{i}$ are energy levels of the cluster).

In the present paper $X^{i j}\left(\vec{R}_{q}\right)$ operators must be built on the complete set of $x$, $y$ and $z$-components of $\vec{S}$. The general form of (2.6) remains unchanged.

For $f_{0}$ particle cluster, the reference system Hamiltonian (2.2) is defined in the $2^{f_{0}}$-component quasispinor basis. The generalized Pauli matrices in this case may be introduced as follows [7]:

$$
\begin{array}{cl}
\sigma_{1}^{\alpha}=S^{\alpha} \times I \times I \times \ldots \times I, & \sigma_{2}^{\alpha}=I \times S^{\alpha} \times I \times \ldots \times I, \\
\vdots & \\
\sigma_{f_{0}-1}^{\alpha}=I \times I \times \ldots \times S^{\alpha} \times I, \quad & \sigma_{f_{0}}^{\alpha}=I \times I \times \ldots \times I \times S^{\alpha},
\end{array}
$$

where $I$ is a $2 \times 2$ unit matrix and $\times$ is a tensor product symbol.

Applying a unitary transformation $W\left(W^{-1} \sigma_{f}^{\alpha} W=\tilde{\sigma}_{f}^{\alpha}\right)$ one can reduce $(2.2)$ into a diagonal form. Then it is convenient to expand $\tilde{\sigma}_{f}^{\alpha}$ in a finite series in $2^{f_{0}}$ component Hubbard-Stasyuk operators $X^{\mu}[6]$

$$
\tilde{\sigma}_{f}^{\alpha}\left(\vec{R}_{q}\right)=\sum_{\mu=1}^{2^{2 f_{0}}} A_{\mu}^{(f, \alpha)} X^{\mu}\left(R_{q}\right)
$$

Here $A_{\mu}^{(f, \alpha)}$ are matrix elements of $\tilde{\sigma}_{f}^{\alpha}$ operators in the (2.8) representation, $\mu$ enumerates the transitions between $i$-th and $j$-th cluster states, $\mu=2^{f_{0}}(i-1)+j$ $\left(i, j=1,2, \ldots 2^{f_{0}}\right)$. The $U$ matrix may be found as a solution of the following set of secular equations:

$$
\sum_{\mu, \mu^{\prime}=1}^{2^{2 f_{0}}}\left\{\sum_{f, f^{\prime}=1}^{f_{0}} \sum_{\alpha} J_{f f^{\prime}}^{\alpha \alpha}\left(\vec{R}_{q}, \vec{R}_{q^{\prime}}\right) A_{\mu}^{(f, \alpha)} A_{\mu^{\prime}}^{\left(f^{\prime}, \alpha\right)} U_{\mu \lambda} U_{\mu^{\prime} \lambda^{\prime}}\right\}=\Phi_{\lambda}\left(\vec{R}_{q}, \vec{R}_{q^{\prime}}\right) \delta_{\lambda \lambda^{\prime}}
$$


As a result, in the generalized transition operators (2.6) representation, one gets a total cluster system Hamiltonian (2.1) in the diagonal in $\lambda$ form:

$$
H=\sum_{\lambda=1}^{2^{2 f_{0}}}\left\{\sum_{q=1}^{N} \Lambda_{\lambda} Y_{\lambda}\left(\vec{R}_{q}\right)-\frac{1}{2} \sum_{q, q^{\prime}=1}^{N} \Phi_{\lambda}\left(\vec{R}_{q}, \vec{R}_{q^{\prime}}\right) Y_{\lambda}\left(\vec{R}_{q}\right) Y_{\lambda}\left(\vec{R}_{q^{\prime}}\right)\right\}
$$

Here

$$
\Lambda_{\lambda}=\sum_{i=1}^{2_{0}} a_{i} U_{i i \lambda}
$$

is the energy of the $\lambda$-th state of the cluster (nominally transformed levels $a_{i}$ ), $\Phi_{\lambda}\left(\vec{R}_{1}, \vec{R}_{q^{\prime}}\right)$ is the $\lambda$-th eigenvalue of the intercluster interaction matrix.

Generalized transition operators (2.6) satisfy the commutation relation:

$$
\begin{aligned}
& {\left[Y_{\lambda}\left(\vec{R}_{q}\right), Y_{\lambda^{\prime}}\left(\vec{R}_{q^{\prime}}\right)\right]=\sum_{\mu} W_{\lambda \lambda^{\prime}}^{\mu} Y_{\lambda}\left(\vec{R}_{q}\right) \delta_{q q^{\prime}},} \\
& W_{\lambda \lambda^{\prime}}^{\mu}=\sum_{r, s, t}\left\{U_{r s \lambda} U_{s t \lambda^{\prime}}-U_{s t \lambda} U_{r s \lambda^{\prime}}\right\} U_{r t \mu},
\end{aligned}
$$

$r, s, t$ are ordinary indices and $\lambda, \mu$ are double indices.

As it was mentioned in the Introduction, in the present paper two types of long ranging interparticle potentials will be regarded. The first one is the Ising-type potential:

$$
J_{f f^{\prime}}^{\alpha \beta}\left(\vec{R}_{q}, \vec{R}_{q^{\prime}}\right)=\left\{\begin{array}{cl}
J_{f f^{\prime}}^{\alpha \alpha}\left(\vec{R}_{q}, \vec{R}_{q^{\prime}}\right), & \alpha=z \\
0, & \alpha=x, y
\end{array}\right.
$$

and the second one is the Heisenberg-type potential:

$$
J_{f f^{\prime}}^{\alpha \beta}\left(\vec{R}_{q}, \vec{R}_{q^{\prime}}\right)=J_{f f^{\prime}}^{\alpha \alpha}\left(\vec{R}_{q}, \vec{R}_{q^{\prime}}\right), \quad \alpha=x, y, z .
$$

The herein proposed method of clusterization of the (2.1) Hamiltonian into the form $(2.11)$ can be easily applied also to the potentials $J_{f f^{\prime}}^{\alpha \beta}\left(\vec{R}_{q}, \vec{R}_{q^{\prime}}\right)$ with other possible relations between their $\alpha, \beta$ components. The central point in such a procedure is the problem of (2.10) secular equations solution.

One can be easily convinced in the fact, that for a usual Heisenberg model (only one spin in a cell, i.e. $\left.f_{0}=1, V=0\right)$ the representation (2.2) is equivalent to (2.11) where:

$$
\begin{gathered}
Y_{1}=\frac{1}{\sqrt{2}}\left(X^{11}+X^{22}\right)=\frac{1}{\sqrt{2}}, \quad Y_{2}=\frac{1}{\sqrt{2}}\left(X^{12}+X^{21}\right)=\sqrt{2} S^{x}, \\
Y_{3}=\frac{1}{\sqrt{2}}\left(X^{12}+X^{21}\right)=\mathrm{i} \sqrt{2} S^{y},
\end{gathered}
$$

and besides

$$
\begin{aligned}
& \left(S^{x}\right)^{2}+\left(S^{4}\right)^{2}+\left(S^{z}\right)^{2}=\frac{3}{4} \\
& X^{11}+X^{22}=1, \quad\left(Y_{1}\right)^{2}+\left(Y_{2}\right)^{2}+\left(Y_{3}\right)^{2}+\left(Y_{4}\right)^{2}=1 .
\end{aligned}
$$


The advantages of $Y_{\lambda}$ operators representation will be demonstared when using them for nontrivial clusters $\left(f_{0} \geqslant 2\right)$. As a real cluster system, a complete investigation of which will be performed in future, we shall regard a system with $f_{0}=2$, that is the one adequate to the $\mathrm{Cu}_{2}\left(\mathrm{CH}_{3} \mathrm{COO}\right)_{4} \cdot 2 \mathrm{H}_{2} \mathrm{O}$ crystal. $\Phi_{\lambda}\left(\vec{R}, \vec{R}^{\prime}\right)$ matrices for Ising and Heisenberg types of interactions in the two-particle cluster system are presented in appendix A. Corresponding $U$ matrices are presented in appendix B. The nonzero coefficients of (2.11) Hamiltonian are:

$$
\begin{aligned}
& \Lambda_{1}=-\frac{V}{\sqrt{2}}, \quad \Lambda_{6}=\frac{3 V}{2}, \quad \Lambda_{11}=-\frac{V}{2}, \quad \Lambda_{16}=-\sqrt{2} h, \\
& \Phi_{7}=J_{11}-J_{12}, \quad \Phi_{16}=J_{11}+J_{12}
\end{aligned}
$$

for long-range interaction of the Ising type and

$$
\begin{aligned}
& \Lambda_{1}=-\frac{V}{\sqrt{2}}, \quad \Lambda_{2}=-\sqrt{2} h, \quad \Lambda_{14}=\frac{3}{2} V, \quad \Lambda_{15}=-\frac{V}{2}, \\
& \Phi_{2}=\Phi_{5}=-\Phi_{11}=J_{11}+J_{12}, \\
& \Phi_{4}=\Phi_{7}=-\Phi_{10}=J_{11}-J_{12}
\end{aligned}
$$

for long range interaction of the Heisenberg type.

The algorithm of clusterization procedure does not depend on the size of clusters, so it can be also performed for the systems with $f_{0}=3,4, \ldots$ in a similar way. Our future task is to investigate the thermodynamic properties of the reference system and to build the functional of partition function for the total system. The latter is necessary for the calculation of thermodynamic functions in the neighbourhood of the phase transition point.

\section{Thermodynamics of the reference two-particle system}

One can see, that Hamiltonian in the form of the reference system, presented by the first term of $(2.11)$

$$
H_{0}=\sum_{q=1}^{N} \sum_{\lambda=1}^{16} \Lambda_{\lambda} Y_{\lambda}\left(\vec{R}_{q}\right)
$$

is not a diagonal one. The reason is that only combinations of $\Lambda_{\lambda}((2.17)$ or $(2.18))$ are true levels of cluster energy. Really, the diagonal is the Hamiltonian:

$$
\begin{aligned}
H_{0}=\sum_{q=1}^{N}\{ & \frac{\Lambda_{1}+\Lambda_{16}}{2}\left(Y_{1}\left(\vec{R}_{q}\right)+Y_{16}\left(\vec{R}_{q}\right)\right)+\Lambda_{6} Y_{6}\left(\vec{R}_{q}\right)+\Lambda_{11} Y_{11}\left(\vec{R}_{q}\right) \\
& \left.+\frac{\Lambda_{1}-\Lambda_{16}}{2}\left(Y_{1}\left(\vec{R}_{q}\right)-Y_{16}\left(\vec{R}_{q}\right)\right)\right\}
\end{aligned}
$$

where $\Lambda_{\lambda}$ are taken from (2.17) (for (2.18) case a simple substitution: $\Lambda_{1} \rightarrow \Lambda_{1}$, $\Lambda_{6} \rightarrow \Lambda_{14}, \Lambda_{11} \rightarrow \Lambda_{15}, \Lambda_{16} \rightarrow \Lambda_{2}$ must be made) 
The partition function $Z_{0}=\operatorname{Tr}\left\{\mathrm{e}^{-\beta H}\right\}$ is equal to

$$
\begin{aligned}
Z_{0} & =Z_{01}^{N}, \\
Z_{01} & =2 \mathrm{e}^{-\beta \frac{\Lambda_{1}}{\sqrt{2}}} \cosh \frac{\beta \Lambda_{16}}{\sqrt{2}}+\mathrm{e}^{-\beta \Lambda_{6}}+\mathrm{e}^{-\beta \Lambda_{11}} \\
& =2 \mathrm{e}^{\beta \frac{V}{2}} \cosh \beta h+\mathrm{e}^{-\frac{3}{2} \beta V}+\mathrm{e}^{\frac{\beta V}{2}} .
\end{aligned}
$$

It is easy now to calculate the average values of $Y_{1}, Y_{6}, Y_{11}, Y_{16}$ operators, using (3.3).

$$
\begin{aligned}
& \left\langle Y_{\lambda}\right\rangle_{0}=-\frac{\partial}{\partial \beta \Lambda_{\lambda}} \ln Z_{01}, \\
& \left\langle Y_{1}\right\rangle_{0}=\frac{\sqrt{2} \mathrm{e}^{-\frac{\beta \Lambda_{1}}{\sqrt{2}}} \cosh \frac{\beta \Lambda_{16}}{\sqrt{2}}}{Z_{01}}, \quad\left\langle Y_{6}\right\rangle_{0}=\frac{\mathrm{e}^{-\beta \Lambda_{6}}}{Z_{01}}, \\
& \left\langle Y_{11}\right\rangle_{0}=\frac{\mathrm{e}^{-\beta \Lambda_{11}}}{Z_{01}}, \quad\left\langle Y_{16}\right\rangle_{0}=-\frac{\sqrt{2} \mathrm{e}^{-\frac{\beta \Lambda_{1}}{\sqrt{2}}} \sinh \frac{\beta \Lambda_{16}}{\sqrt{2}}}{Z_{01}} .
\end{aligned}
$$

Here $\beta=1 / k T, k$ is the Boltzmann constant, $T$ is the absolute temperature.

Thermodynamic functions of the reference system are calculated based on the free energy:

$$
F_{0}=-\frac{1}{\beta} \ln Z_{0}=-\frac{N}{\beta} \ln \left\{\mathrm{e}^{-\frac{3 \beta V}{2}}+\mathrm{e}^{\frac{\beta V}{2}}(1+2 \cosh \beta h)\right\} .
$$

For entropy $(S)$, internal energy $(U)$, heat capacity $\left(C_{v}\right)$, magnetic moment $(M)$ and magnetic susceptibility $(\chi)$ per one cell, one can obtain the following formulae:

$$
\begin{aligned}
& S=-\left(\frac{\partial F_{0}}{\partial T}\right)_{h}=N k \ln \left\{\mathrm{e}^{-\frac{-3 \beta V}{2}}+\mathrm{e}^{\frac{\beta V}{2}}(1+2 \cosh \beta h)\right\} \\
& +N \frac{3 \beta V-\beta V \mathrm{e}^{2 \beta V}(1+2 \cosh \beta h)-4 \beta h \mathrm{e}^{2 \beta V} \sinh \beta h}{2\left[1+\mathrm{e}^{2 \beta V}(1+2 \cosh \beta h)\right]}, \\
& U=F_{0}+T S=N \frac{3 V-V \mathrm{e}^{2 \beta V}(1+2 \cosh \beta h)-4 h \sinh \beta h}{2\left[1+\mathrm{e}^{2 \beta V}(1+2 \cosh \beta h)\right]}, \\
& C_{v}=\left(\frac{\partial u}{\partial T}\right)_{v}=N k \beta^{2} \mathrm{e}^{2 \beta V}\left\{\frac{V^{2}(1+2 \cosh \beta h)+5 h V \sinh \beta h+2 h^{2} \sinh \beta h}{1+\mathrm{e}^{2 \beta V}(1+2 \cosh \beta h)}\right. \\
& \left.+\frac{\left[3 V-V(1+2 \cosh \beta h) \mathrm{e}^{2 \beta V}-4 h \mathrm{e}^{2 \beta V} \sinh \beta h\right][V(1+2 \cosh \beta h)-h \sinh \beta h]}{\left[1+\mathrm{e}^{2 \beta h}(1+2 \cosh \beta V)\right]^{2}}\right\}, \\
& M=-\frac{1}{N}\left(\frac{\partial F_{0}}{\partial h}\right)_{T}=\frac{2 \mathrm{e}^{2 \beta V} \sinh \beta h}{1+\mathrm{e}^{2 \beta V}(1+2 \cosh \beta h)}, \\
& \chi=\frac{1}{N}\left(\frac{\partial^{2} F_{0}}{\partial h^{2}}\right)_{T}=\beta\left\{\frac{2 \mathrm{e}^{2 \beta V} \cosh \beta h}{1+\mathrm{e}^{2 \beta V}(1+2 \cosh \beta h)}-\left[\frac{2 \mathrm{e}^{2 \beta V} \sinh \beta h}{1+\mathrm{e}^{2 \beta V}(1+2 \cosh \beta h)}\right]^{2}\right\} .
\end{aligned}
$$


The total magnetic moment of one cluster, from another point of view, is determined by the average value of $z$-component of both spins. In accordance with (2.7) and with appendix B.1, one can get:

$$
M\left(\vec{R}_{q}\right)=\left\langle\sigma_{1}^{z}\left(\vec{R}_{q}\right)+\sigma_{2}^{z}\left(\vec{R}_{q}\right)\right\rangle=\sqrt{2}\left\langle Y_{16}\left(\vec{R}_{q}\right)\right\rangle
$$

Thus, only one component of generalized transition operators, namely $Y_{16}$, is responsible for the formation of the total magnetic moment of the cluster. This component may be called the "active" one is the phase transition process. A similar definition was first introduced in [7]. If $\left\langle Y_{16}\left(\vec{R}_{q}\right)\right\rangle$ did not depend on the number of a cell $q$, then(3.7) describes a uniform order in crystal, i.e. a ferromagnetic state. All other possible arrangements of clusters (antiferromagnetic, ferrimagnetic, spiral, incommensurate and so on) are completely determined by the spatial distribution of $\left\langle Y_{16}\left(\vec{R}_{q}\right)\right\rangle$. It must be noted, that after averaging in $(3.7)$ with the $H_{0}$ Hamiltonian, the obtained $M(\vec{R})$ coinsides with $M$ from (3.6).

Taking into account that at low temperature region the internal effective field proportional to magnetization appears, so $h \rightarrow \tilde{h}$

$$
\tilde{h}=h+\frac{\Phi_{16}(0)}{2} M
$$

and the expression for $M(3.6)$ transforms into equation

$$
M=\frac{2 \sinh \beta\left(h+\frac{\Phi_{16}(0)}{2} M\right)}{1+\mathrm{e}^{-2 \beta V}+2 \cosh \beta\left(h+\frac{\Phi_{16}(0)}{2} M\right)},
$$

one may find the asymptotic behaviour of thermodynamic functions:

$$
\begin{aligned}
& \lim _{T \rightarrow 0} S=0, \quad \lim _{T \rightarrow 0} C_{v}=0, \quad \lim _{T \rightarrow 0} \chi=0, \\
& \lim _{T \rightarrow 0} M=1, \quad(V>0), \quad \lim _{T \rightarrow 0} M \simeq 1 \quad(V<0) .
\end{aligned}
$$

It may be tested that the first line of the expressions (3.10) did not depend on the sign of $V$ (attractive, or repulsive interaction between particles in the cluster). So, the thermodynamic stability of the investigated cluster reference system is not violated.

For a consistent solution of the problem of thermodynamics of the total cluster system (2.11) it is necessary to use the methods, which are effective in the phase transition point neighbourhood. In the present paper a modified method of collective variables, which takes into account a structural peculiarity and interparticle interaction potential properties of the investigated system, is used. The first step in this way is the construction of the functional of the partition function. 


\section{Functional of the partition function}

The general approach to the construction of the functional of the partition function for cluster systems was developed in $[6,7]$. Since those systems possess quantum properties, to calculate the statistical operator $\mathrm{e}^{-\beta H}$ it was necessary to use the interaction representation with respect to a reference system Hamiltonian. So, collective variables and all coefficients of the functional became dependent on Matsubara's frequecies.

Because the physical system considered in the present paper is described by a quantum Heisenberg-like Hamiltonian, the main features of its functional of the partition function are similar to the ones obtained in $[7,10]$. The principal difference in the form of functional coefficients is determined by the properties of the reference system. The latter one determines the values of cumulant averages of generalized transition operators products which form the above mentioned coefficients. The calculation of those cumulants is now a central point of consideration.

In the collective variables representation, the total functional of partition function of the interacting cluster system is $[10,11]$ :

$$
\begin{aligned}
Z= & Z_{0} \int\left(\mathrm{d} \rho_{\lambda}(\vec{k}, \nu)\right)^{N} \prod_{\lambda} \prod_{k \leqslant B} \prod_{\nu} J\left(\rho_{\lambda},(\vec{k}, \nu)\right) \\
& \times \exp \left\{\frac{\beta}{2} \sum_{\lambda} \sum_{k \leqslant B} \sum_{\nu} \Phi_{\lambda}(\vec{k}) \rho_{\lambda}(\vec{k}, \nu) \rho_{\lambda}(-\vec{k},-\nu)\right\} .
\end{aligned}
$$

Here $\rho_{\lambda}(\vec{k}, \nu)$ is a collective variable corresponding to the generalized transition operator (operator of a cluster state) in the frequency-momentum representation:

$$
\begin{aligned}
\hat{\rho}_{\lambda}(\vec{k}, \nu) & =\frac{1}{\sqrt{\beta}} \int_{0}^{\beta} \mathrm{d} \beta^{\prime} \mathrm{e}^{-\mathrm{i} \beta^{\prime} \nu} \sum_{q=1}^{N} \mathrm{e}^{-\beta^{\prime} H_{0}} Y_{\lambda}\left(\vec{R}_{q}\right) \mathrm{e}^{\beta^{\prime} H_{0}} \mathrm{e}^{\mathrm{i} \vec{k} \vec{R}_{q}} \\
\Phi_{\lambda}(\vec{k}) & =\sum_{q} \Phi_{\lambda}\left(\vec{R}_{q}\right) \mathrm{e}^{\mathrm{i} \vec{k} R_{q}}
\end{aligned}
$$

is a Fourier transform of the $\lambda$-th eigenvalue of the intercluster interaction potential, $\nu$ is the Matsubara's frequency.

The transition Jacobian from the set of generalized transition operators $\hat{\rho}_{\lambda}(\vec{k}, \nu)$ to the collective variables $\rho_{\lambda}(\vec{k}, \nu)$

$$
J\left(\rho_{\lambda}(\vec{k}, \nu)\right)=\operatorname{Tr} \prod_{\lambda, k, \nu} \delta\left(\rho_{\lambda}(\vec{k}, \nu)-\hat{\rho}_{\lambda}(\vec{k}, \nu)\right),
$$

as it usually takes place in the collective variables method [5], we present in the exponential form:

$$
J\left(\rho_{\lambda},(\vec{k}, \nu)\right)=\int\left(\mathrm{d} \omega_{\lambda}(\vec{k}, \nu)\right)^{N} \exp \left\{\mathrm{i} 2 \pi \sum_{\lambda} \sum_{k \leqslant B} \sum_{\nu} \omega_{\lambda}(\vec{k}, \nu) \rho_{\lambda}(\vec{k}, \nu)\right\}
$$




$$
\begin{aligned}
\times \exp \{ & \sum_{n=1}^{\infty} \frac{(-\mathrm{i} 2 \pi)^{n}}{n !} \sum_{\lambda_{1}, k_{1}, \nu_{1}} \ldots \sum_{\lambda_{n}, k_{n}, \nu_{n}} \mathcal{M}_{\lambda_{1} \ldots \lambda_{n}}\left(\vec{k}_{1}, \nu_{1}, \ldots, \vec{k}_{n}, \nu_{n}\right) \\
& \left.\times \omega_{\lambda_{1}}\left(\vec{k}_{1}, \nu_{1}\right) \ldots \omega_{\lambda_{n}}\left(\vec{k}_{n}, \nu_{n}\right)\right\} .
\end{aligned}
$$

Cumulants $\mathcal{M}_{\lambda_{1} \ldots \lambda_{n}}\left(\vec{k}_{1}, \nu_{1}, \ldots, \vec{k}_{n}, \nu_{n}\right)$ must be calculated as functional derivations [11]:

$$
\begin{aligned}
& \mathcal{M}_{\lambda_{1} \ldots \lambda_{n}}\left(\vec{k}_{1}, \nu_{1}, \ldots, \vec{k}_{n}, \nu_{n}\right)= \\
& \quad=\left.\frac{\partial^{4}}{\partial \omega_{\lambda_{1}}\left(\vec{k}_{1}, \nu_{1}\right) \ldots \partial \omega_{\lambda_{n}}\left(\vec{k}_{n}, \nu_{n}\right)} \ln \left\langle T \exp \left\{\sum_{\lambda} \sum_{k, \nu} \omega_{\lambda}(\vec{k}, \nu) \hat{\rho}_{\lambda}(\vec{k}, \nu)\right\}\right\rangle\right|_{0},
\end{aligned}
$$

where $\omega_{\lambda}(\vec{k}, \nu)$ is a variable conjugated to $\rho_{\lambda}(\vec{k}, \nu), T$ is a symbol for "time" arrangement with respect to the inverse temperature $\beta, Z_{0}$ is the (3.3) expressions,

$$
\langle\ldots\rangle_{0}=\operatorname{Tr}\left[\ldots \mathrm{e}^{-\beta H_{0}}\right]\left\{\operatorname{Tr}\left[\mathrm{e}^{-\beta H_{0}}\right]\right\}^{-1},
$$

$H_{0}$ being determined by $(3.1)$.

The functional integrals (4.1) (4.5) contain all powers of collective variables $\rho_{\lambda}(\vec{k}, \nu)$. Therefore, it is impossible to integrate (4.1) over $\rho_{\lambda}(\vec{k}, \nu)$ analitically in an obvious form. Usually, for analitic or computer calculations, the limit expressions, which include only the second, the fourth or some higher powers of $\rho_{\lambda}(\vec{k}, \nu)$ are used. The only requirement must be satisfied: all the expressions obtained after the integration remain undivergent at any temperature and at arbitrary values of parameters of the Hamiltonian. Such a distribution is called a basic one. In general, it has not been proved that the use of more complicated distributions (taking into account additional higher powers of $\rho(k)$ in the exponent form of (4.5)) leads to a better mathematical convergence of the obtained physical results. Most probably, the choise of a concrete form of basic distribution determines a definite statistical model. As regards the physical system in a phase transition point neighbourhood, the simplest basic distribution is a quartic distribution. Such functionals are usually called the Ginzburg-Landau functionals. In the present paper only Ginzburg-Landau functionals will be regarded. It must be noted that due to the external field $h$ existence, the quartic basic distribution containes all powers of collective variables (even and odd ones) to the fourth inclusive. The basic distribution determines the basic measure density of collective variables near the phase transition point. Cumulants (4.5) are the coefficients of this measure density form.

It should be also noted that an additional problem, concerning the functional integrals convergence, is the infinite number of collective variables $\rho_{\lambda}(\vec{k}, \nu)$ due to $\vec{k}$ and $\nu$ quasi-continuous nature. But taking into account that in the phase transition point neighbourhood only the variables with small $\vec{k}$ (large distancies of correlations) and small $\nu$ (a finite rather large $T_{c}$ for real ferromagnets) play an essential role, from the physical consideration one may suppose the role of large $\vec{k}$ and lange $\nu$ to 
be negligibly small. So, for physical models of ferromagnetic phase transitions, the regarded functional integrals (4.1), (4.2) or (4.4) are finite.

To obtain a cumulant average value of a product of the arbitrary numbers of generalized transition operators, the expressions (2.6), (4.6) and appendix B will be used. Because collective variables describe only the long-range part of interaction (short-range part of interaction is completely included into $H_{0}$ ), among all possible cumulant average quantities we are interested in those for which $\Phi_{\lambda}\left(\vec{R}_{q}, \vec{R}_{q^{\prime}} \neq 0\right)$, i.e. $\lambda=7,16$ for intercluster interactions of the Ising type (see (2.17)) and $\lambda=$ $2,4,5,7,10,11$ for intercluster interactions of the Heisenberg type (see (2.18)). In the interaction representation, the Hubbard operator

$$
X^{i j}\left(\vec{R}_{q}, \beta^{\prime}\right)=\mathrm{e}^{-\beta^{\prime} H_{0}} X^{i j}\left(\vec{R}_{q}\right) \mathrm{e}^{\beta^{\prime} H_{0}}
$$

satisfies a very important relation [6]:

$$
R_{0} X^{i j}\left(\vec{R}_{q}, \beta^{\prime}\right)=\mathrm{e}^{-\beta \lambda_{i j}} X^{i j}\left(\vec{R}_{q}, \beta^{\prime}\right) R_{0},
$$

where

$$
R_{0}=\mathrm{e}^{-\beta H_{0}}
$$

is a statistical operator of the reference system, and

$$
\lambda_{i j}=E_{i}-E_{j}
$$

is a distance between energy levels of the cluster. When executing a cyclic commutation of the $X^{i j}\left(\vec{R}_{q}, \beta^{\prime}\right)$ operator under the Tr symbol and taking into account (4.9), one can prove:

$$
\left\langle X^{i_{1} j_{1}}\left(\vec{R}_{q_{1}}, \beta_{1}\right) X^{i_{2} j_{2}}\left(\vec{R}_{q_{2}}, \beta_{2}\right) X^{i_{3} j_{3}}\left(\vec{R}_{q_{3}}, \beta_{3}\right) \ldots\right\rangle=0
$$

at $\lambda_{i_{1} j_{1}}+\lambda_{i_{2} j_{2}}+\lambda_{i_{3} j_{3}}+\ldots \neq 0$.

This relation simplifies the procedure of calculation because it shows the expressions which are identically equal to zero.

Based on the (2.6), (4.6) and (4.2) for $n$-th order cumulant one obtains a formula:

$$
\begin{aligned}
& \mathcal{M}_{\lambda_{1} \lambda_{2} \ldots \lambda_{n}}\left(\vec{k}_{1}, \nu_{1}, \vec{k}_{2}, \nu_{2}, \ldots \vec{k}_{n}, \nu_{n}\right)= \\
& =\frac{1}{N^{n / 2}} \sum_{q_{1}, q_{2}, \ldots q_{n}=1} \exp \left\{-\mathrm{i}\left[\vec{k}_{1} \vec{R}_{q_{1}}+\vec{k}_{2} \vec{R}_{q_{2}}+\ldots+\vec{k}_{n} \vec{R}_{q_{n}}\right]\right\} \\
& \quad \times \frac{1}{\beta^{n}} \int_{0}^{\beta} \mathrm{d} \beta_{1} \int_{0}^{\beta} \mathrm{d} \beta_{2} \ldots \int_{0}^{\beta} \mathrm{d} \beta_{n} \exp \left\{\mathrm{i}\left[\beta_{1} \nu_{1}+\beta_{2} \nu_{2}+\ldots+\beta_{n} \nu_{n}\right]\right\} \\
& \quad \times \sum_{i_{1}, j_{1}, i_{2}, j_{2}, \ldots i_{n}, j_{n}} U_{i_{1} j_{1} \lambda_{1}} U_{i_{2} j_{2} \lambda_{2}} \ldots U_{i_{n} j_{n} \lambda_{n}} \\
& \quad \times\left\langle T X^{i_{1} j_{1}}\left(\vec{R}_{q_{1}} \beta_{1}\right) X^{i_{2} j_{2}}\left(\vec{R}_{q_{2}} \beta_{2}\right) \ldots X^{i_{n} j_{n}}\left(\vec{R}_{q_{n}} \beta_{n}\right)\right\rangle_{0}^{\mathrm{c}} .
\end{aligned}
$$

Here $\langle\ldots\rangle_{0}^{c}$ means a cumulant average. To calculate this cumulant average quantity, the Block-Wick-Dominicis theorem [12] in the form [13] may be used. It must be noted that all the operators $X^{i j}\left(\vec{R}_{q}\right)$ in (4.13) are of Bose type because the total member of particles in every cell is constant.

Since for Ising and Heisenberg types of intercluster interactions, the complete sets of cumulants are different, those sets will be presented separately. 


\subsection{Ising-type intercluster interactions}

For generalized transition operators

$$
\begin{aligned}
& Y_{7}=\frac{1}{\sqrt{2}}\left[X^{23}+X^{32}\right], \\
& Y_{16}=\frac{1}{\sqrt{2}}\left[X^{11}-X^{44}\right]
\end{aligned}
$$

the total set of nonzero cumulant averages to the fourth order inclusive is as follows.

For the first-order cumulants:

$$
\begin{aligned}
& \mathcal{M}_{7}^{\text {Ising }}(\vec{k}, \nu)=0 \\
& \mathcal{M}_{16}^{\text {Ising }}(\vec{k}, \nu)=\frac{\sqrt{2} \mathrm{e}^{\beta \frac{V}{2}} \sinh \beta h}{Z_{0}} \delta(\vec{k}) \delta(\nu) ;
\end{aligned}
$$

for the second-order cumulants:

$$
\begin{aligned}
& \mathcal{M}_{77}^{\text {Ising }}\left(\vec{k}_{1}, \nu_{1}, \vec{k}_{2}, \nu_{2}\right)=\frac{4 V \mathrm{e}^{-\beta \frac{V}{2}} \sinh \beta V}{\beta\left(4 V^{2}+\nu^{2}\right) Z_{0}} \delta\left(\vec{k}_{1}+\vec{k}_{2}\right) \delta\left(\nu_{1}+\nu_{2}\right) \\
& \mathcal{M}_{1616}^{\text {Ising }}\left(\vec{k}_{1}, \nu_{1}, \vec{k}_{2}, \nu_{2}\right)=\left[\frac{\mathrm{e}^{\beta \frac{V}{2}} \cosh \beta h}{Z_{0}}-\frac{2 \mathrm{e}^{\beta V} \sinh ^{2} \beta h}{Z_{0}^{2}}\right] \delta\left(\vec{k}_{1}+\vec{k}_{2}\right) \delta\left(\nu_{i}\right) \\
& \mathcal{M}_{716}^{\text {Ising }}\left(\vec{k}_{1}, \nu_{1}, \vec{k}_{2}, \nu_{2}\right)=0
\end{aligned}
$$

for the third-order cumulants:

$$
\begin{aligned}
& \mathcal{M}_{777}^{\text {Ising }}\left(\vec{k}_{1}, \nu_{1}, \vec{k}_{2}, \nu_{2}, \vec{k}_{3}, \nu_{3}\right)=0, \\
& \mathcal{M}_{161616}^{\text {Ising }}\left(\vec{k}_{1}, \nu_{1}, \vec{k}_{2}, \nu_{2}, \vec{k}_{3}, \nu_{3}\right)=\frac{\mathrm{e}^{\beta \frac{V}{2}} \sinh \beta h}{\sqrt{2} Z_{0}} \delta\left(\vec{k}_{1}+\vec{k}_{2}+\vec{k}_{3}\right) \delta\left(\nu_{i}\right) \\
& \quad-\frac{3 \sqrt{2} \mathrm{e}^{\beta V} \cosh \beta h \sinh \beta h}{Z_{0}^{2}} \delta\left(\vec{k}_{1}+\vec{k}_{2}\right) \delta\left(\vec{k}_{3}\right) \delta\left(\nu_{i}\right)+\frac{4 \sqrt{2} \mathrm{e}^{\frac{3}{2} \beta V} \sinh ^{3} \beta h}{Z_{0}^{3}} \delta\left(\vec{k}_{i}\right) \delta\left(\nu_{i}\right), \\
& \mathcal{M}_{7716}^{\text {Ising }}\left(\vec{k}_{1}, \nu_{1}, \vec{k}_{2}, \nu_{2}, \vec{k}_{3}, \nu_{3}\right)=-\frac{4 \sqrt{2} V \sinh \beta V \sinh \beta h}{\beta\left(4 V^{2}+\nu^{2}\right) Z_{0}^{2}} \\
& \quad \times \delta\left(\vec{k}_{1}+\vec{k}_{2}\right) \delta\left(\vec{k}_{3}\right) \delta\left(\nu_{1}+\nu_{2}\right) \delta\left(\nu_{3}\right), \\
& \mathcal{M}_{71616}^{\text {Ising }}\left(\vec{k}_{1}, \nu_{1}, \vec{k}_{2}, \nu_{2}, \vec{k}_{3}, \nu_{3}\right)=0 ;
\end{aligned}
$$

for the fourth-order cumulants:

$$
\begin{aligned}
\mathcal{M}_{7777}^{\text {Ising }}\left(\vec{k}_{1}, \nu_{1}, \vec{k}_{2}, \nu_{2}, \vec{k}_{3}, \nu_{3}, \vec{k}_{4}, \nu_{4}\right)= \\
=\frac{4 V \mathrm{e}^{-\beta \frac{V}{2}} \sinh \beta V}{2 \beta\left(4 V^{2}+\nu^{2}\right) Z_{0}} \delta\left(\vec{k}_{1}+\vec{k}_{2}+\vec{k}_{3}+\vec{k}_{4}\right) \delta\left(\nu_{1}+\nu_{2}+\nu_{3}+\nu_{4}\right) \\
\quad-\frac{48 V^{2} \mathrm{e}^{-\beta V} \sinh ^{2} \beta V}{\beta^{2}\left(4 V^{2}+\nu^{2}\right)^{2} Z_{0}^{2}} \delta\left(\vec{k}_{1}+\vec{k}_{2}\right) \delta\left(\vec{k}_{3}+\vec{k}_{4}\right) \delta\left(\nu_{1}+\nu_{2}\right) \delta\left(\nu_{3}+\nu_{4}\right),
\end{aligned}
$$




$$
\begin{aligned}
\mathcal{M}_{16}^{\text {Ising }}{ }_{16}{ }_{16}\left(\vec{k}_{1}, \nu_{1}, \vec{k}_{2}, \nu_{2}, \vec{k}_{3}, \nu_{3}, \vec{k}_{4}, \nu_{4}\right)=\frac{\mathrm{e}^{\beta \frac{V}{2}} \cosh \beta h}{2 Z_{0}} \delta\left(\vec{k}_{1}+\vec{k}_{2}+\vec{k}_{3}+\vec{k}_{4}\right) \delta\left(\nu_{i}\right) \\
\quad-\frac{4 \mathrm{e}^{\beta V} \sinh ^{2} \beta h}{Z_{0}^{2}} \delta\left(\vec{k}_{1}+\vec{k}_{2}+\vec{k}_{3}\right) \delta\left(\vec{k}_{4}\right) \delta\left(\nu_{i}\right) \\
\quad-\frac{3 \mathrm{e}^{\beta V} \cosh ^{2} \beta h}{Z_{0}^{2}} \delta\left(\vec{k}_{1}+\vec{k}_{2}\right) \delta\left(\vec{k}_{3}+\vec{k}_{4}\right) \delta\left(\nu_{i}\right) \\
\quad+\frac{24 \mathrm{e}^{3 \beta \frac{V}{2}} \cosh \beta h \sinh ^{2} \beta h}{Z_{0}^{3}} \delta\left(\vec{k}_{1}+\vec{k}_{2}\right) \delta\left(\vec{k}_{3}\right) \delta\left(\vec{k}_{4}\right) \delta\left(\nu_{i}\right) \\
\quad-\frac{24 \mathrm{e}^{2 \beta V} \sinh ^{4} \beta h}{Z_{0}^{4}} \delta\left(\vec{k}_{i}\right) \delta\left(\nu_{i}\right), \\
\mathcal{M}_{771616}^{\text {Ising }}\left(\vec{k}_{1}, \nu_{1}, \vec{k}_{2}, \nu_{2}, \vec{k}_{3}, \nu_{3}, \vec{k}_{4}, \nu_{4}\right)= \\
=-\frac{4 V \sinh \beta V \cosh \beta h}{\beta\left(4 V^{2}+\nu^{2}\right) Z_{0}^{2}} \delta\left(\vec{k}_{1}+\vec{k}_{2}\right) \delta\left(\vec{k}_{3}+\nu_{3}\right) \delta\left(\nu_{1}+\nu_{2}\right) \delta\left(\nu_{3}\right) \delta\left(\nu_{4}\right) \\
\quad+\frac{8 V \mathrm{e}^{\beta \frac{V}{2}} \sinh \beta V \sinh ^{2} \beta h}{\beta\left(4 V^{2}+\nu^{2}\right) Z_{0}^{3}} \delta\left(\vec{k}_{1}+\nu_{2}\right) \delta\left(\vec{k}_{3}\right) \delta\left(\vec{k}_{4}\right) \delta\left(\nu_{1}+\nu_{2}\right) \delta\left(\nu_{3}\right) \delta\left(\nu_{4}\right),
\end{aligned}
$$

all the rest cumulants of this order are equal to zero.

One may verify that at $h=0$ (external field is absent) all odd-order cumulants in (4.15)-(4.18) are equal to zero. Other interesting properties of the obtained cumulants must be noted. Only cumulants built on the $\hat{\rho}_{7}(\vec{k}, \nu)$ operators are dependent on Matsubara's frequencies. But all the cumulants built solely on the $\hat{\rho}_{16}(\vec{k}, \nu)$ operators, are independent on $\nu$. Let's remind that collective variables $\hat{\rho}_{16}(\vec{k}, \nu)$ are responsible for the appearance of a long-range order in the physical cluster system because $\left\langle\hat{\rho}_{16}(0,0)\right\rangle \sim\left\langle Y_{16}\left(\vec{R}_{q}\right)\right\rangle$ (see (2.17), (3.4) and (3.7)) determines a total spin moment of the cluster and its mean value is proportional to the external magnetic field intensity. So, it confirms a statement that ferromagnetic ordering (phase transition) is an essentially classical phenomenon [15]. At the same time, the considered system possesses quantum properties, which are important at low temperatires when basic state effects dominate.

The asymptotic behaviour of cumulants at $h \rightarrow 0, V \rightarrow 0$ are quite different for $\nu=0$ and for $\nu \neq 0$ :

$$
\begin{array}{ll}
\nu=0 & \mathcal{M}_{77}^{\text {Ising }}=\mathcal{M}_{1616}^{\text {Ising }}=\frac{1}{4}, \quad \mathcal{M}_{7777}^{\text {Ising }}=\mathcal{M}_{16161616}^{\text {Ising }}=-\frac{1}{16}, \\
\nu \neq 0 & \text { all cumulants are equal to zero. }
\end{array}
$$

Taking into account that at $V=0, h=0$, the partition function of the isolated cluster is twice bigger than the partition function of one spin, as well as taking into account the normalizing factors in (4.8), one may pass from the expressions (4.24) to classical spin cumulants of Ising model (see [4]):

$$
\begin{aligned}
& \mathcal{M}_{77}^{\text {Ising }}=\mathcal{M}_{1616}^{\text {Ising }}=\frac{1}{4} \cdot 2 \cdot(\sqrt{2})^{2}=1 \\
& \mathcal{M}_{7777}^{\text {Ising }}=\mathcal{M}_{16161616}^{\text {Ising }}=\frac{1}{8} \cdot 2 \cdot(\sqrt{2})^{4}-\frac{3}{16} \cdot 2^{2} \cdot(\sqrt{2})^{4}=-2 .
\end{aligned}
$$




\subsection{Heisenberg-type intercluster interactions}

For generalized transition operators

$$
\begin{aligned}
Y_{2}\left(\vec{R}_{q}\right) & =\frac{1}{\sqrt{2}}\left[X^{11}\left(\vec{R}_{q}\right)-X^{44}\left(\vec{R}_{q}\right)\right], \\
Y_{4}\left(\vec{R}_{q}\right) & =\frac{1}{2}\left[X^{12}\left(\vec{R}_{q}\right)+X^{21}\left(\vec{R}_{q}\right)-X^{24}\left(\vec{R}_{q}\right)-X^{42}\left(\vec{R}_{q}\right)\right], \\
Y_{5}\left(\vec{R}_{q}\right) & =\frac{1}{2}\left[X^{13}\left(\vec{R}_{q}\right)+X^{31}\left(\vec{R}_{q}\right)+X^{34}\left(\vec{R}_{q}\right)+X^{43}\left(\vec{R}_{q}\right)\right], \\
Y_{7}\left(\vec{R}_{q}\right) & =\frac{1}{\sqrt{2}}\left[X^{23}\left(\vec{R}_{q}\right)+X^{32}\left(\vec{R}_{q}\right)\right], \\
Y_{10}\left(\vec{R}_{q}\right) & =\frac{1}{2}\left[-X^{13}\left(\vec{R}_{q}\right)+X^{31}\left(\vec{R}_{q}\right)-X^{34}\left(\vec{R}_{q}\right)+X^{43}\left(\vec{R}_{q}\right)\right], \\
Y_{11}\left(\vec{R}_{q}\right) & =\frac{1}{2}\left[-X^{12}\left(\vec{R}_{q}\right)+X^{21}\left(\vec{R}_{q}\right)+X^{24}\left(\vec{R}_{q}\right)-X^{42}\left(\vec{R}_{q}\right)\right]
\end{aligned}
$$

the mean value of which forms in this case a total set of cumulants, we have: for the first-order cumulant:

$$
\mathcal{M}_{2}^{\text {Haiz. }}(\vec{k}, \nu)=\mathcal{M}_{16}^{\text {Ising }}(\vec{k}, \nu),
$$

for the second-order cumulants:

$$
\begin{aligned}
& \mathcal{M}_{22}^{\text {Haiz. }}\left(\vec{k}_{1}, \nu_{1}, \vec{k}_{2}, \nu_{2}\right)=\mathcal{M}_{1616}^{\text {Ising }}\left(\vec{k}_{1}, \nu_{1}, \vec{k}_{2}, \nu_{2}\right) \\
& \mathcal{M}_{44}^{\text {Haiz. }}\left(\vec{k}_{1}, \nu_{1}, \vec{k}_{2}, \nu_{2}\right)=-\mathcal{M}_{1010}^{\text {Haiz. }}\left(\vec{k}_{1}, \nu_{1}, \vec{k}_{2}, \nu_{2}\right) \\
& =\frac{\mathrm{e}^{\beta \frac{V}{2}}}{2 \beta Z_{0}}\left[\frac{(2 V+h)\left(\mathrm{e}^{\beta h}-\mathrm{e}^{-2 \beta V}\right)}{(2 V+h)^{2}+\nu^{2}}+\frac{(2 V-h)\left(\mathrm{e}^{-\beta h}-\mathrm{e}^{-2 \beta V}\right)}{(2 V-h)^{2}+\nu^{2}}\right] \\
& \quad \times \delta\left(\vec{k}_{1}+\vec{k}_{2}\right) \delta\left(\nu_{1}+\nu_{2}\right), \\
& \mathcal{M}_{55}^{\text {Haiz. }}\left(\vec{k}_{1}, \nu_{1}, \vec{k}_{2}, \nu_{2}\right)=-\mathcal{M}_{1111}^{\text {Haiz. }}\left(\vec{k}_{1}, \nu_{1}, \vec{k}_{2}, \nu_{2}\right) \\
& =\frac{\mathrm{e}^{\beta \frac{V}{2}} h \sinh \beta h}{\beta\left(h^{2}+\nu^{2}\right) Z_{0}} \delta\left(\vec{k}_{1}+\vec{k}_{2}\right) \delta\left(\nu_{1}+\nu_{2}\right), \\
& \mathcal{M}_{77}^{\text {Haiz. }}\left(\vec{k}_{1}, \nu_{1}, \vec{k}_{2}, \nu_{2}\right)=-\mathcal{M}_{77}^{\text {Ising }}\left(\vec{k}_{1}, \nu_{1}, \vec{k}_{2}, \nu_{2}\right) ;
\end{aligned}
$$

for the third-order cumulants:

$$
\begin{aligned}
& \mathcal{M}_{222}^{\text {Haiz. }}\left(\vec{k}_{1}, \nu_{1}, \vec{k}_{2}, \nu_{2}, \vec{k}_{3}, \nu_{3}\right)=\mathcal{M}_{161616}^{\text {Ising }}\left(\vec{k}_{1}, \nu_{1}, \vec{k}_{2}, \nu_{2}, \vec{k}_{3}, \nu_{3}\right), \\
& \mathcal{M}_{244}^{\text {Haiz. }}\left(\vec{k}_{1}, \nu_{1}, \vec{k}_{2}, \nu_{2}, \vec{k}_{3}, \nu_{3}\right)=-\mathcal{M}_{21010}^{\text {Haiz. }}\left(\vec{k}_{1}, \nu_{1}, \vec{k}_{2}, \nu_{2}, \vec{k}_{3}, \nu_{3}\right) \\
& =-\frac{\mathrm{e}^{\beta V} \sinh \beta h}{\sqrt{2} \beta Z_{0}^{2}}\left[\frac{(2 V+h)\left(\mathrm{e}^{\beta h}-\mathrm{e}^{-2 \beta V}\right)}{(2 V+h)^{2}+\nu^{2}}+\frac{(2 V-h)\left(\mathrm{e}^{-\beta h}-\mathrm{e}^{-2 \beta V}\right)}{(2 V-h)^{2}+\nu^{2}}\right] \\
& \quad \times \delta\left(\vec{k}_{1}\right) \delta\left(\vec{k}_{2}+\vec{k}_{3}\right) \delta\left(\nu_{1}\right) \delta\left(\nu_{2}+\nu_{3}\right), \\
& \mathcal{M}_{255}^{\text {Haiz. }}\left(\vec{k}_{1}, \nu_{1}, \vec{k}_{2}, \nu_{2}, \vec{k}_{3}, \nu_{3}\right)=-\mathcal{M}_{21111}^{\text {Haiz. }}\left(\vec{k}_{1}, \nu_{1}, \vec{k}_{2}, \nu_{2}, \vec{k}_{3}, \nu_{3}\right)
\end{aligned}
$$




$$
\begin{gathered}
=-\frac{\sqrt{2} \mathrm{e}^{\beta V} h \sinh ^{2} \beta h}{\beta\left(h^{2}+\nu^{2}\right) Z_{0}^{2}} \delta\left(\vec{k}_{1}\right) \delta\left(\vec{k}_{2}+\vec{k}_{3}\right) \delta\left(\nu_{1}\right) \delta\left(\nu_{2}+\nu_{3}\right), \\
\mathcal{M}_{772}^{\text {Haiz. }}\left(\vec{k}_{1}, \nu_{1}, \vec{k}_{2}, \nu_{2}, \vec{k}_{3}, \nu_{3}\right)=\mathcal{M}_{7716}^{\text {Ising }}\left(\vec{k}_{1}, \nu_{1}, \vec{k}_{2}, \nu_{2}, \vec{k}_{3}, \nu_{3}\right) ;
\end{gathered}
$$

for the fourth-order cumulants:

$$
\begin{aligned}
& \mathcal{M}_{2222}^{\text {Haiz. }}\left(\vec{k}_{1}, \nu_{1}, \vec{k}_{2}, \nu_{2}, \vec{k}_{3}, \nu_{3}, \vec{k}_{4}, \nu_{4}\right)=\mathcal{M}_{16161616}^{\text {Ising }}\left(\vec{k}_{1}, \nu_{1}, \vec{k}_{2}, \nu_{2}, \vec{k}_{3}, \nu_{3}, \vec{k}_{4}, \nu_{4}\right) \text {, } \\
& \mathcal{M}_{5555}^{\text {Haiz. }}\left(\vec{k}_{1}, \nu_{1}, \vec{k}_{2}, \nu_{2}, \vec{k}_{3}, \nu_{3}, \vec{k}_{4}, \nu_{4}\right)=\mathcal{M}_{11111111}^{\text {Haiz. }}\left(\vec{k}_{1}, \nu_{1}, \vec{k}_{2}, \nu_{2}, \vec{k}_{3}, \nu_{3}, \vec{k}_{4}, \nu_{4}\right) \\
& =\frac{\mathrm{e}^{\beta \frac{V}{2}} h \sinh \beta h}{2 \beta\left(h^{2}+\nu^{2}\right) Z_{0}} \delta\left(\vec{k}_{1}+\vec{k}_{2}+\vec{k}_{3}+\vec{k}_{4}\right) \delta\left(\nu_{1}+\nu_{2}+\nu_{3}+\nu_{4}\right) \\
& -\frac{3 \mathrm{e}^{\beta V} h^{2} \sinh ^{2} \beta h}{\beta^{2}\left(h^{2}+\nu^{2}\right)^{2} Z_{0}^{2}} \delta\left(\vec{k}_{1}+\vec{k}_{2}\right) \delta\left(\vec{k}_{3}+\vec{k}_{4}\right) \delta\left(\nu_{1}+\nu_{2}\right) \delta\left(\nu_{3}+\nu_{4}\right), \\
& \mathcal{M}_{4444}^{\text {Haiz. }}\left(\vec{k}_{1}, \nu_{1}, \vec{k}_{2}, \nu_{2}, \vec{k}_{3}, \nu_{3}, \vec{k}_{4}, \nu_{4}\right)=\mathcal{M}_{10101010}^{\text {Haiz. }}\left(\vec{k}_{1}, \nu_{1}, \vec{k}_{2}, \nu_{2}, \vec{k}_{3}, \nu_{3}, \vec{k}_{4}, \nu_{4}\right) \\
& =\frac{\mathrm{e}^{\beta \frac{V}{2}}}{4 \beta Z_{0}}\left[\frac{(2 V+h)\left(\mathrm{e}^{\beta h}-\mathrm{e}^{-2 \beta V}\right)}{(2 V+h)^{2}+\nu^{2}}+\frac{(2 V-h)\left(\mathrm{e}^{-\beta h}-\mathrm{e}^{-2 \beta V}\right)}{(2 V-h)^{2}+\nu^{2}}\right] \\
& \times \delta\left(\vec{k}_{1}+\vec{k}_{2}+\vec{k}_{3}+\vec{k}_{3}\right) \delta\left(\nu_{1}+\nu_{2}+\nu_{3}+\nu_{4}\right) \\
& -\frac{3 \mathrm{e}^{\beta V}}{4 \beta^{2} Z_{0}^{2}}\left[\frac{(2 V+h)\left(\mathrm{e}^{\beta h}-\mathrm{e}^{-2 \beta V}\right)}{(2 V+h)^{2}+\nu^{2}}+\frac{(2 V-h)\left(\mathrm{e}^{-\beta h}-\mathrm{e}^{-2 \beta V}\right)}{(2 V-h)^{2}+\nu^{2}}\right]^{2} \\
& \times \delta\left(\vec{k}_{1}+\vec{k}_{2}\right) \delta\left(\vec{k}_{3}+\vec{k}_{4}\right) \delta\left(\nu_{1}+\nu_{2}\right) \delta\left(\nu_{3}+\nu_{4}\right), \\
& \mathcal{M}_{7777}^{\text {Haiz. }}\left(\vec{k}_{1}, \nu_{1}, \vec{k}_{2}, \nu_{2}, \vec{k}_{3}, \nu_{3}, \vec{k}_{4}, \nu_{4}\right)=\mathcal{M}_{7777}^{\text {Ising }}\left(\vec{k}_{1}, \nu_{1}, \vec{k}_{2}, \nu_{2}, \vec{k}_{3}, \nu_{3}, \vec{k}_{4}, \nu_{4}\right) \text {, } \\
& \mathcal{M}_{2255}^{\text {Haiz. }}\left(\vec{k}_{1}, \nu_{1}, \vec{k}_{2}, \nu_{2}, \vec{k}_{3}, \nu_{3}, \vec{k}_{4}, \nu_{4}\right)=-\mathcal{M}_{221111}^{\text {Haiz. }}\left(\vec{k}_{1}, \nu_{1}, \vec{k}_{2}, \nu_{2}, \vec{k}_{3}, \nu_{3}, \vec{k}_{4}, \nu_{4}\right) \\
& =\frac{\mathrm{e}^{\beta \frac{V}{2}}}{4 Z_{0}}\left[\frac{2 h \sinh \beta h}{\beta\left(h^{2}+\nu^{2}\right)}-1\right] \delta\left(\vec{k}_{1}+\vec{k}_{2}+\vec{k}_{3}+\vec{k}_{3}+\vec{k}_{4}\right) \delta\left(\nu_{1}+\nu_{2}+\nu_{3}+\nu_{4}\right) \\
& -\frac{\mathrm{e}^{\beta V} h \cosh \beta h \sinh \beta h}{\beta\left(h^{2}+\nu^{2}\right) Z_{0}^{2}} \delta\left(\vec{k}_{1}+\vec{k}_{2}\right) \delta\left(\vec{k}_{3}+\vec{k}_{4}\right) \delta\left(\nu_{1}\right) \delta\left(\nu_{2}\right) \delta\left(\nu_{3}+\nu_{4}\right) \\
& +\frac{2 \mathrm{e}^{3 \beta \frac{V}{2}} h \sinh ^{3} \beta h}{\beta\left(h^{2}+\nu^{2}\right) Z_{0}^{3}} \delta\left(\vec{k}_{1}\right) \delta\left(\vec{k}_{2}\right) \delta\left(\vec{k}_{3}+\vec{k}_{4}\right) \delta\left(\nu_{1}\right) \delta\left(\nu_{2}\right) \delta\left(\nu_{3}+\nu_{4}\right), \\
& \mathcal{M}_{2244}^{\text {Haiz. }}\left(\vec{k}_{1}, \nu_{1}, \vec{k}_{2}, \nu_{2}, \vec{k}_{3}, \nu_{3}, \vec{k}_{4}, \nu_{4}\right)=-\mathcal{M}_{221010}^{\text {Haiz. }}\left(\vec{k}_{1}, \nu_{1}, \vec{k}_{2}, \nu_{2}, \vec{k}_{3}, \nu_{3}, \vec{k}_{4}, \nu_{4}\right) \\
& =\frac{\mathrm{e}^{\beta \frac{V}{2}}}{4 Z_{0}}\left[\frac{(2 V+h)\left(\mathrm{e}^{\beta h}-\mathrm{e}^{-2 \beta V}\right)}{\beta\left((2 V+h)^{2}+\nu^{2}\right)}+\frac{(2 V-h)\left(\mathrm{e}^{-\beta h}-\mathrm{e}^{-2 \beta V}\right)}{\beta\left((2 V-h)^{2}+\nu^{2}\right)}-\mathrm{e}^{-2 \beta V}\right] \\
& \times \delta\left(\vec{k}_{1}+\vec{k}_{2}+\vec{k}_{3}+\vec{k}_{4}\right) \delta\left(\nu_{1}+\nu_{2}+\nu_{3}+\nu_{4}\right) \\
& -\frac{\mathrm{e}^{\beta V} \cosh \beta h}{2 \beta Z_{0}^{2}}\left[\frac{(2 V+h)\left(\mathrm{e}^{\beta h}-\mathrm{e}^{-2 \beta V}\right)}{(2 V+h)^{2}+\nu^{2}}+\frac{(2 V-h)\left(\mathrm{e}^{-\beta h}-\mathrm{e}^{-2 \beta V}\right)}{(2 V-h)^{2}+\nu^{2}}\right] \\
& \times \delta\left(\vec{k}_{1}+\vec{k}_{2}\right) \delta\left(\vec{k}_{3}+\vec{k}_{4}\right) \delta\left(\nu_{1}\right) \delta\left(\nu_{2}\right) \delta\left(\nu_{3}+\nu_{4}\right) \\
& +\frac{\mathrm{e}^{3 \beta \frac{V}{2}} \sinh ^{2} \beta h}{\beta Z_{0}^{3}}\left[\frac{(2 V+h)\left(\mathrm{e}^{\beta h}-\mathrm{e}^{-2 \beta V}\right)}{(2 V+h)^{2}+\nu^{2}}+\frac{(2 V-h)\left(\mathrm{e}^{-\beta h}-\mathrm{e}^{-2 \beta V}\right)}{(2 V-h)^{2}+\nu^{2}}\right]
\end{aligned}
$$




$$
\begin{aligned}
& \times \delta\left(\vec{k}_{1}\right) \delta\left(\vec{k}_{2}\right) \delta\left(\vec{k}_{3}+\vec{k}_{4}\right) \delta\left(\nu_{1}\right) \delta\left(\nu_{2}\right) \delta\left(\nu_{3}+\nu_{4}\right), \\
& \mathcal{M}_{551111}^{\text {Haiz. }}\left(\vec{k}_{1}, \nu_{1}, \vec{k}_{2}, \nu_{2}, \vec{k}_{3}, \nu_{3}, \vec{k}_{4}, \nu_{4}\right)= \\
& =-\frac{\mathrm{e}^{\beta \frac{V}{2}}}{4 Z_{0}} \delta\left(\vec{k}_{1}+\vec{k}_{2}+\vec{k}_{3}+\vec{k}_{4}\right) \delta\left(\nu_{1}+\nu_{2}+\nu_{3}+\nu_{4}\right) \\
& +\frac{\mathrm{e}^{\beta V} h^{2} \sinh ^{2} \beta h}{\beta^{2}\left(h^{2}+\nu^{2}\right) Z_{0}^{2}} \delta\left(\vec{k}_{1}+\vec{k}_{2}\right) \delta\left(\vec{k}_{3}+\vec{k}_{4}\right) \delta\left(\nu_{1}+\nu_{2}\right) \delta\left(\nu_{3}+\nu_{4}\right) ; \\
& \mathcal{M}_{5577}^{\text {Haiz. }}\left(\vec{k}_{1}, \nu_{1}, \vec{k}_{2}, \nu_{2}, \vec{k}_{3}, \nu_{3}, \vec{k}_{4}, \nu_{4}\right)=-\mathcal{M}_{111177}^{\text {Haiz. }}\left(\vec{k}_{1}, \nu_{1}, \vec{k}_{2}, \nu_{2}, \vec{k}_{3}, \nu_{3}, \vec{k}_{4}, \nu_{4}\right) \\
& =\frac{\mathrm{e}^{\beta \frac{V}{2}}}{4 Z_{0}} \delta\left(\vec{k}_{1}+\vec{k}_{2}+\vec{k}_{3}+\vec{k}_{4}\right) \delta\left(\nu_{1}+\nu_{2}+\nu_{3}+\nu_{4}\right) \\
& -\frac{4 V h \sinh \beta V \sinh \beta h}{\beta^{2}\left(4 h^{2}+\nu^{2}\right)\left(h^{2}+\nu^{2}\right) Z_{0}^{2}} \delta\left(\vec{k}_{1}+\vec{k}_{2}\right) \delta\left(\vec{k}_{3}+\vec{k}_{4}\right) \delta\left(\nu_{1}+\nu_{2}\right) \delta\left(\nu_{3}+\nu_{4}\right), \\
& \mathcal{M}_{551010}^{\text {Haiz. }}\left(\vec{k}_{1}, \nu_{1}, \vec{k}_{2}, \nu_{2}, \vec{k}_{3}, \nu_{3}, \vec{k}_{4}, \nu_{4}\right)=-\mathcal{M}_{111144}^{\text {Haiz. }}\left(\vec{k}_{1}, \nu_{1}, \vec{k}_{2}, \nu_{2}, \vec{k}_{3}, \nu_{3}, \vec{k}_{4}, \nu_{4}\right) \\
& =-\frac{\mathrm{e}^{\beta \frac{V}{2}}}{4 Z_{0}}\left[\frac{2 h \sinh \beta h}{\beta\left(h^{2}+\nu^{2}\right)}-1\right] \delta\left(\vec{k}_{1}+\vec{k}_{2}+\vec{k}_{3}+\vec{k}_{4}\right) \delta\left(\nu_{1}+\nu_{2}+\nu_{3}+\nu_{4}\right) \\
& +\frac{\mathrm{e}^{\beta V} h \sinh \beta h}{2 \beta^{2}\left(h^{2}+\nu^{2}\right) Z_{0}^{2}}\left[\frac{(2 V+h)\left(\mathrm{e}^{\beta h}-\mathrm{e}^{-2 \beta V}\right)}{(2 V+h)^{2}+\nu^{2}}+\frac{(2 V-h)\left(\mathrm{e}^{-\beta h}-\mathrm{e}^{-2 \beta V}\right)}{(2 V-h)^{2}+\nu^{2}}\right] \\
& \times \delta\left(\vec{k}_{1}+\vec{k}_{2}\right) \delta\left(\vec{k}_{3}+\vec{k}_{4}\right) \delta\left(\nu_{1}+\nu_{2}\right) \delta\left(\nu_{3}+\nu_{4}\right), \\
& \mathcal{M}_{4477}^{\text {Haiz. }}\left(\vec{k}_{1}, \nu_{1}, \vec{k}_{2}, \nu_{2}, \vec{k}_{3}, \nu_{3}, \vec{k}_{4}, \nu_{4}\right)=-\mathcal{M}_{771010}^{\text {Haiz. }}\left(\vec{k}_{1}, \nu_{1}, \vec{k}_{2}, \nu_{2}, \vec{k}_{3}, \nu_{3}, \vec{k}_{4}, \nu_{4}\right) \\
& =\frac{\mathrm{e}^{-\beta V}}{4 Z_{0}} \delta\left(\vec{k}_{1}+\vec{k}_{2}+\vec{k}_{3}+\vec{k}_{4}\right) \delta\left(\nu_{1}+\nu_{2}+\nu_{3}+\nu_{4}\right) \\
& -\frac{2 V \sinh \beta V}{\beta^{2}\left(4 V^{2}+\nu^{2}\right) Z_{0}^{2}}\left[\frac{(2 V+h)\left(\mathrm{e}^{\beta h}-\mathrm{e}^{-2 \beta V}\right)}{(2 V+h)^{2}+\nu^{2}}+\frac{(2 V-h)\left(\mathrm{e}^{-\beta h}-\mathrm{e}^{-2 \beta V}\right)}{(2 V-h)^{2}+\nu^{2}}\right] \\
& \times \delta\left(\vec{k}_{1}+\vec{k}_{2}\right) \delta\left(\vec{k}_{3}+\vec{k}_{4}\right) \delta\left(\nu_{1}+\nu_{2}\right) \delta\left(\nu_{3}+\nu_{4}\right), \\
& \mathcal{M}_{441010}^{\text {Haiz. }}\left(\vec{k}_{1}, \nu_{1}, \vec{k}_{2}, \nu_{2}, \vec{k}_{3}, \nu_{3}, \vec{k}_{4}, \nu_{4}\right)= \\
& =-\frac{\mathrm{e}^{-\beta V}}{4 Z_{0}} \delta\left(\vec{k}_{1}+\vec{k}_{2}+\vec{k}_{3}+\vec{k}_{4}\right) \delta\left(\nu_{1}+\nu_{2}+\nu_{3}+\nu_{4}\right) \\
& +\frac{\mathrm{e}^{\beta V}}{4 \beta^{2} Z_{0}^{2}}\left[\frac{(2 V+h)\left(\mathrm{e}^{\beta h}-\mathrm{e}^{-2 \beta V}\right)}{(2 V+h)^{2}+\nu^{2}}+\frac{(2 V-h)\left(\mathrm{e}^{-\beta h}-\mathrm{e}^{-2 \beta V}\right)}{(2 V-h)^{2}+\nu^{2}}\right] \\
& \times \delta\left(\vec{k}_{1}+\vec{k}_{2}\right) \delta\left(\vec{k}_{3}+\vec{k}_{4}\right) \delta\left(\nu_{1}+\nu_{2}\right) \delta\left(\nu_{3}+\nu_{4}\right) \text {. }
\end{aligned}
$$

All the rest Heisenberg-type cumulants up to the fourth-order inclusive are equal to zero.

Clearly, the higher-order cumulants for the both types of intercluster interactions considered here can be also obtained using the formula (4.13). Moreover, this formula is suitable for other different possible types of interparticle interactions.

So, the complete sets of two-particle cluster system cumulants for two types of intercluster interactions (Ising's and Heisenberg's ones) are found. The functional of 
partition function is completely defined. Its final form is as follows:

$$
\begin{aligned}
Z=Z_{0} \int\left(\mathrm{d} \rho_{\lambda}(\vec{k}, \nu)\right)^{N} \exp \left\{\sum_{\lambda} \sum_{k, \nu} \frac{\beta}{2} \Phi_{\lambda}(\vec{k}) \rho_{\lambda}(\vec{k}, \nu) \rho_{\lambda}(-\vec{k},-\nu)\right\} \\
\times \int\left(\mathrm{d} \omega_{\lambda}(\vec{k}, \nu)\right)^{N} \exp \left\{\mathrm{i} 2 \pi \sum_{\lambda} \sum_{k, \nu}\left[\rho_{\lambda}(\vec{k}, \nu)-\mathcal{M}_{\lambda}(\vec{k}, \nu)\right] \omega_{\lambda}(\vec{k}, \nu)\right. \\
-\frac{(2 \pi)^{2}}{2} \sum_{\lambda_{1}, \lambda_{2}} \sum_{k, \nu} \mathcal{M}_{\lambda_{1} \lambda_{2}}(\vec{k}, \nu,-\vec{k},-\nu) \omega_{\lambda_{1}}(\vec{k}, \nu) \omega_{\lambda_{2}}(-\vec{k},-\nu) \\
+\frac{\mathrm{i}(2 \pi)^{3}}{3 !} \sum_{\lambda_{1}, \lambda_{2}, \lambda_{3}} \sum_{\substack{k_{1}, k_{2}, k_{3} \\
\nu_{1}, \nu_{2}, \nu_{3}}} \mathcal{M}_{\lambda_{1} \lambda_{2} \lambda_{3}}\left(\vec{k}_{1}, \nu_{1}, \vec{k}_{2}, \nu_{2}, \vec{k}_{3}, \nu_{3}\right) \\
+\frac{(2 \pi)^{4}}{4 !} \sum_{\lambda_{1} \lambda_{2}, \lambda_{3}, \lambda_{4}} \sum_{\substack{k_{1}, k_{2}, k_{3}, k_{4} \\
\nu_{1}, \nu_{2}, \nu_{3}, \nu_{4}}} \mathcal{M}_{\lambda_{1} \lambda_{2} \lambda_{3} \lambda_{4}}\left(\vec{k}_{1}, \nu_{1}, \vec{k}_{2}, \nu_{2}, \vec{k}_{3}, \nu_{3}, \vec{k}_{4}, \nu_{4}\right) \\
\times \omega_{\lambda_{2}}\left(\vec{k}_{2}, \nu_{2}\right) \omega_{\lambda_{3}}\left(\vec{k}_{3}, \nu_{3}\right) \\
\left.\times \omega_{\lambda_{1}}\left(\vec{k}_{1}, \nu_{1}\right) \omega_{\lambda_{2}}\left(\vec{k}_{2}, \nu_{2}\right) \omega_{\lambda_{3}}\left(\vec{k}_{3}, \nu_{3}\right) \omega_{\lambda_{4}}\left(\vec{k}_{4}, \nu_{4}\right)\right\} .
\end{aligned}
$$

For practical calculation of the expression (4.26), the layer by layer integration over collective variables $\rho_{\lambda}(\vec{k}, \nu)$, similar to those exploited in $[10,14]$, may be used. This problem is a subject of another paper.

\section{Conclusions}

1. The Hamiltonian of the cluster magnetic system (2.1) includes two essentially non-equivalent parts of interparticle interactions. The first one describes intracluster interactions between spin's particles and may be represented by exchange Heisenberg form. The second one is responsible for long-range intercluster interactions of a dipole-dipole type. Depending on of the physical nature of the investigated systems this part of interaction may be described by Ising or by Heisenberg types of Hamiltonians.

2. The problem of complete determination of quantum states and corresponding energy levels of a basic cluster system may be solved using generalized transition operators $Y_{\lambda}\left(\vec{R}_{q}\right)$. Those operators are similar to the well-known Hubbard-Stasyuk operators $X^{i j}\left(\vec{R}_{q}\right)$, but their form essentially depends on the structure and on interparticle interaction peculiarity of the system studied.

3. To construct the functional of the partition function of a quantum magnetic system, the collective variables method is proposed. The coefficients of this functional are expressed by the cluster cumulants, which are calculated for both Ising and Heisenberg types of intercluster interactions. Among different 
sorts of collective variables only certain ones are responsible for ferro-, antiferromagnetic or other kinds of the magnetic ordering. It has been shown that "ferromagnetic-type" collective variables behave like the classical ones (they don't depend on Matsubara frequencies). Hence, the corresponding phase transition may be treated as a classical phenomenon. 


\section{A. Intercluster interaction matrix for two-particle cluster system}

\section{A.1. Ising-type interactions}

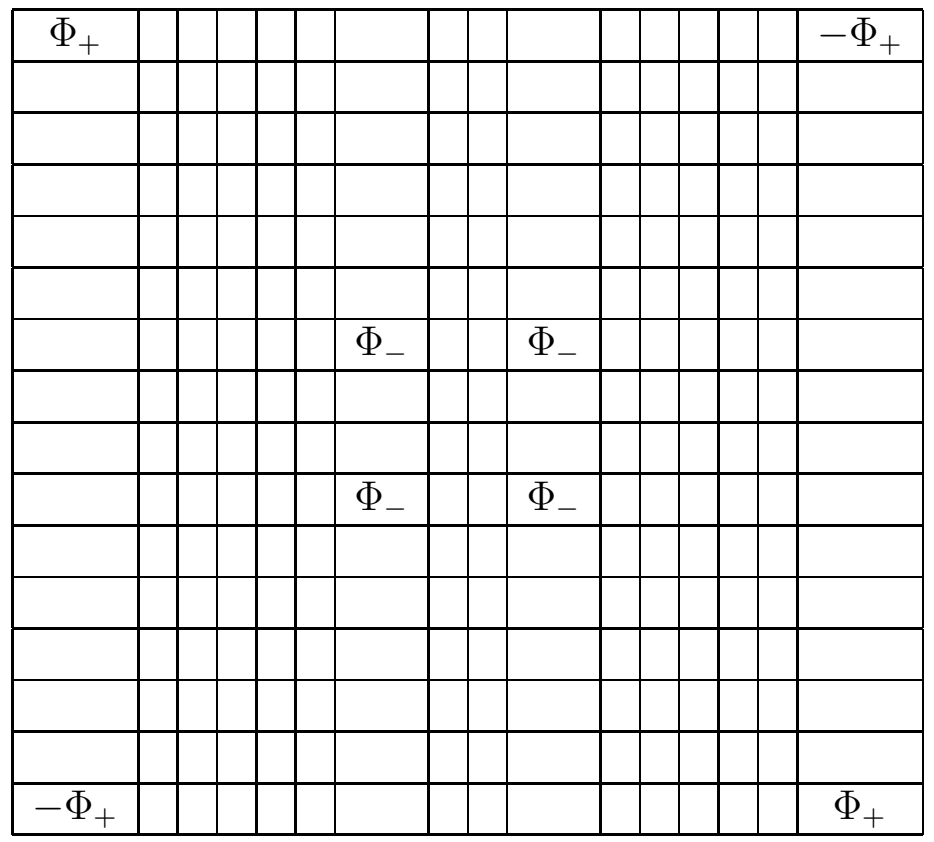

\section{A.2. Heisenberg-type interactions}

\begin{tabular}{|c|c|c|c|c|c|c|c|c|c|c|c|}
\hline$\Phi_{+}$ & & & & & & & & & & & $-\Phi_{+}$ \\
\hline & & & $\Phi_{-}$ & & & & & & $-\Phi_{-}$ & & \\
\hline & & & & & & $\Phi_{+}$ & & & & $\Phi_{+}$ & \\
\hline & & & & & & & & & & & \\
\hline & $\Phi_{-}$ & & & & $-\Phi_{-}$ & & & & & & \\
\hline & & & & & & & & & & & \\
\hline & & & & $\Phi$ & & & $\Phi$ & & & & \\
\hline & & & $-\Phi_{-}$ & & & & & & $\Phi_{-}$ & & \\
\hline & & $\overline{\Phi_{+}}$ & & & & & & $\Phi_{+}$ & & & \\
\hline & & & & $\Phi$ & & & $\Phi$ & & & . & \\
\hline & & & & & & & & & & & \\
\hline & & & & & & $\Phi_{+}$ & & & & $\Phi_{+}$ & \\
\hline & & & & & & & & & & & \\
\hline & $-\Phi_{-}$ & & & & $\Phi_{-}$ & & & & & & \\
\hline & & $\overline{\Phi_{+}}$ & & & & & & $\Phi_{+}$ & & & \\
\hline$-\Phi_{+}$ & & & & & & & & & & & $\Phi_{+}$ \\
\hline
\end{tabular}

$$
\Phi_{+}=\frac{1}{2}\left(J_{11}\left(\vec{R}_{q}, \vec{R}_{q^{\prime}}\right)+J_{12}\left(\vec{R}_{q}, \vec{R}_{q^{\prime}}\right)\right), \quad \Phi_{-}=\frac{1}{2}\left(J_{11}\left(\vec{R}_{q}, \vec{R}_{q^{\prime}}\right)-J_{12}\left(\vec{R}_{q}, \vec{R}_{q^{\prime}}\right)\right) .
$$




\section{B. Unitary transfornation matrix $U$ for two-particle cluster system}

\section{B.1. Ising-type interactions}

\begin{tabular}{|c|c|c|c|c|c|c|c|c|c|c|c|c|c|c|c|}
\hline$\frac{1}{\sqrt{2}}$ & & & & & & & & & & & & & & & $\frac{1}{\sqrt{2}}$ \\
\hline & 1 & & & & & & & & & & & & & & \\
\hline & & $\overline{1}$ & & & & & & & & & & & & & \\
\hline & & & 1 & & & & & & & & & & & & \\
\hline & & & & 1 & & & & & & & & & & & \\
\hline & & & & & & & & & & & & & & & \\
\hline & & & & & & $\frac{1}{\sqrt{2}}$ & & & $\frac{1}{\sqrt{2}}$ & & & & & & \\
\hline & & & & & & & 1 & & & & & & & & \\
\hline & & & & & & & & 1 & & & & & & & \\
\hline & & & & & & $\frac{1}{\sqrt{2}}$ & & & $-\frac{1}{\sqrt{2}}$ & & & & & & \\
\hline & & & & & & & & & & 1 & & & & & \\
\hline & & & & & & & & & & & 1 & & & & \\
\hline & & & & & & & & & & & & 1 & & & \\
\hline & & & & & & & & & & & & & 1 & & \\
\hline & & & & & & & & & & & 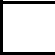 & & & 1 & \\
\hline$\frac{1}{\sqrt{2}}$ & & & & & & & & & & & & & & & $-\frac{1}{\sqrt{2}}$ \\
\hline
\end{tabular}

\section{B.2. Heisenberg-type interactions}

\begin{tabular}{|c|c|c|c|c|c|c|c|c|c|c|c|c|c|c|}
\hline$\frac{\sqrt{2}}{2}$ & $\frac{1}{\sqrt{2}}$ & & & & & & & & & & & & & \\
\hline & & $\frac{1}{2}$ & $\frac{1}{2}$ & & & & & $-\frac{1}{2}$ & $-\frac{1}{2}$ & & & & & \\
\hline & & & & $\frac{1}{2}$ & $\frac{1}{2}$ & & & & & $-\frac{1}{2}$ & $-\frac{1}{2}$ & & & \\
\hline & & & & & & & & & & & & 1 & & \\
\hline & & $\frac{1}{2}$ & $\frac{1}{2}$ & & & & & $\frac{1}{2}$ & $\frac{1}{2}$ & & & & & \\
\hline & & & & & & & & & & & & & & \\
\hline & & & & & & $\frac{1}{\sqrt{2}}$ & $\frac{1}{\sqrt{2}}$ & & & & & & & \\
\hline & & $\frac{1}{2}$ & $-\frac{1}{2}$ & & & & & $-\frac{1}{2}$ & $\frac{1}{2}$ & & & & & \\
\hline & & & & $\frac{1}{2}$ & $\frac{1}{2}$ & & & & & $\frac{1}{2}$ & $\frac{1}{2}$ & & & \\
\hline & & & & & . & $\frac{1}{\sqrt{2}}$ & $-\frac{1}{\sqrt{2}}$ & & & & & & & \\
\hline & & & & & & & & & & & & & 1 & \\
\hline & & & & $\frac{1}{2}$ & $-\frac{1}{2}$ & & & & & $-\frac{1}{2}$ & $\frac{1}{2}$ & & & \\
\hline & & & & & & & & & & & 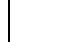 & & & 1 \\
\hline & & $\frac{1}{2}$ & $-\frac{1}{2}$ & & & & & $\frac{1}{2}$ & $-\frac{1}{2}$ & & & & & \\
\hline & & & & $\frac{1}{2}$ & $-\frac{1}{2}$ & & & & & $\frac{1}{2}$ & $-\frac{1}{2}$ & & & \\
\hline & & & & & & & & & & & & & & \\
\hline
\end{tabular}




\section{References}

1. Smart J.S. Effective Field Theories of Magnetism. Moscow, Mir, 1968 (in Russian).

2. Pietraszko A., Lukaszewicz K., Kirpichnikova L.F. // Polish J. Chem., 1995, vol. 69. p. 922.

3. Kuriata J., Lipinski I.E., Korynevskii N.A., Bodziony T. // Physica B, 2001, vol. 307, p. 203.

4. Akhiezer A.I., Bar'yakhtar V.G., Peletminskii S.V. Spin Waves. Moskow, Nauka, 1967 (in Russian).

5. Yukhnovskii I.R. Phase Transition of the Second Order. Collective Variables Method. Singapore, World Scientific, 1987.

6. Korynevskii N.A. // Theor. Math. Phys., 1983, vol. 55, p.291 (in Russian).

7. Yukhnovskii I.R., Korynevskii N.A. // Phys. stat. sol. (b), 1989, vol. 153, p. 583.

8. Hubbard J. // Proc. Roy. Soc., 1965, vol. A285, p. 542.

9. Didukh L.D., Stasyuk I.V. // Phys. Met. Metalloved., 1968, vol. 26, p. 582.

10. Korynevskii N.A. // Ferroelectrics, 1997, vol. 192, p. 45.

11. Korynevskii N.A. The Functional Representation of Partition Function of Quantum Cluster Systems. Preprint of the Institute for Condensed Matter Physics, ICMP-9806U, Lviv, 1998, 31 p. (in Ukrainian).

12. Abrikosov A.A., Gor'kov L.P., Dzialoshynskii I.E. Methods of Quantum Field Theory in Satistic Physics. Moskow. Phys.-Math. Lit., 1962 (in Russian)

13. Slobodyan P.M., Stasyuk I.V. // Theor. Math. Phys., 1974, vol. 19, p. 423 (in Russian)

14. Yukhnovskii I.R., Korynevskii N.A. // Phys. stat. sol. (b), 1989, vol. 154, p. 519.

15. Ma S. Modern Theory of Critical Phenomenon. Moskow, Mir, 1980 (in Russian). 


\section{Про функціональне зображення статистичної суми квантової магнітної кластерної системи}

\section{М.А.Кориневський ${ }^{1,2}$}

1 Інститут фізики конденсованих систем НАН України, 79011 Львів, вул. Свєнціцького, 1

2 Інститут фізики Щецінського університету, Польща, 70451 щецін, вул. Вєлькопольська, 15

Отримано 6 лютого 2002 р., в остаточному вигляді - 13 червня 2002 p.

Обговорюється проблема функціонального зображення для систем, що містять групи атомів з нескомпенсованим спіновим моментом (магнітні кластери). Для зображення функціонала статистичної суми використано варіант методу колективних змінних з виділеною "системою відліку", яка відповідає нульовому наближенню. В якості системи відліку розглядається сукупність ізольованих кластерів. Внутрікластерні взаємодії описуються обмінним гамільтоніаном гайзенбергівського типу, вигляд міжкластерних взаємодій визначається структурою системи, що досліджується. Завдяки використанню попередньо впроваджених узагальнених операторів переходу (близьких до відомих операторів Хаббарда-Стасюка) отримано явну форму функціонала статистичної суми.

Ключові слова: кластерна система, функціонал статистичної суми, система відліку

PACS: 05.60. $+W, 05.70 . \mathrm{Ln}, 05.20 . \mathrm{Dd}, 52.25 . \mathrm{Dg}, 52.25 . \mathrm{Fi}$ 